\title{
Enhanced recovery versus conventional care in gastric cancer surgery: a meta-analysis of randomized and non-randomized controlled trials
}

\author{
Ian Jun Yan Wee ${ }^{1,2} \cdot$ Nicholas Li-Xun Syn ${ }^{2,3} \cdot$ Asim Shabbir ${ }^{2,3} \cdot$ Guowei Kim² $\cdot$ Jimmy B. Y. So ${ }^{2,3}$
}

Received: 7 November 2018 / Accepted: 4 February 2019 / Published online: 25 February 2019

(c) The International Gastric Cancer Association and The Japanese Gastric Cancer Association 2019

\begin{abstract}
Introduction Enhanced recovery after surgery (ERAS) protocols have been successfully integrated into peri-operative management of different cancer surgeries such as colorectal cancer. Their value for gastric cancer surgery, however, remains uncertain.

Methods A search for randomized and observational studies comparing ERAS versus conventional care in gastric cancer surgery was performed according to PRISMA guidelines. Random-effects meta-analyses with inverse variance weighting were conducted, and quality of included studies was assessed using the Cochrane risk-of-bias tool and Newcastle-Ottawa scale (PROSPERO: CRD42017080888).

Results Twenty-three studies involving 2686 patients were included. ERAS was associated with reduced length of hospital stay (WMD -2.47 days, $95 \% \mathrm{CI}-3.06$ to $-1.89, P<0.00001$ ), time to flatus (WMD-0.70 days, $95 \% \mathrm{CI}-1.02$ to -0.37 , $P<0.0001$ ), and hospitalization costs (WMD-USD\$ 4400, 95\% CI - USD $\$ 5580$ to - USD\$3210, $P<0.00001$ ), with consistent results across open and laparoscopic surgery. Postoperative morbidity and 30-day mortality were similar, although a higher rate of readmission was observed in the ERAS group ( $\mathrm{RR}=1.95,95 \% \mathrm{CI} 1.03-3.67, P=0.04)$. Patients in the ERAS arm had significantly attenuated C-reactive protein levels on days 3/4 and 7, interleukin-6 levels on days 1 , and 3/4, and tumor necrosis factor- $\alpha$ levels on days $3 / 4$ postoperatively.

Conclusion Compared to conventional care, ERAS reduces hospital stay, costs, surgical stress response and time to return of gut function, without increasing post-operative morbidity in gastric cancer surgery. However, precaution is necessary to reduce the increased risk of hospital readmission when adopting ERAS.
\end{abstract}

Keywords ERAS $\cdot$ Enhanced recovery after surgery $\cdot$ Gastric surgery $\cdot$ Gastric cancer

\section{Introduction}

Ian Jun Yan Wee and Nicholas Li-Xun Syn contributed equally.

Electronic supplementary material The online version of this article (https://doi.org/10.1007/s10120-019-00937-9) contains supplementary material, which is available to authorized users.

Jimmy B. Y. So

jimmy_so@nuhs.edu.sg

1 Yong Loo Lin School of Medicine, National University of Singapore, Singapore, Singapore

2 Division of General Surgery, University Surgical Cluster, National University Hospital, Singapore, Singapore

3 Department of Surgery, Yong Loo Lin School of Medicine, National University of Singapore, National University Health System, NUHS Tower Block, Level 8, 1E Kent Ridge Road, Singapore 119228, Singapore
Gastric cancer is the fourth leading cause of cancer-related mortality globally, accounting for over 750,000 deaths annually [1]. Surgical resection remains the pillar of gastric cancer management, but is associated with significant postoperative morbidity and healthcare costs [2], hence warranting improvements in surgical standards. First described by Bardram et al. in 1995 [3], the enhanced recovery after surgery (ERAS) pathway adopts a multidisciplinary approach to expedite recovery, alleviate surgical stress response, and reduce complication rates. The ERAS program amalgamates distinct key measures including preoperative counselling; provision of carbohydrate loading prior to surgery; perioperative management of body temperature; early removal of urinary catheters, early postoperative feeding and mobilization. Important procedures that are avoided include bowel 
preparation; premedication; preoperative fasting; perioperative fluid overload; use of drains and nasogastric decompression tubes $[4,5]$.

ERAS programs have long been established in colorectal cancer surgery, demonstrating improved complication rates, reduced length of hospital stay without compromising patient safety [4]. Recently, the enhanced recovery after surgery $\left(\right.$ ERAS $\left.^{\circledR}\right)$ Society published a consensus guideline on gastrectomy for cancer patients [6]. However, the measure of impact following the adoption of these guidelines is yet to be established. Nonetheless, reports evaluating ERAS programs for gastrectomy for gastric cancer are emerging, albeit few in numbers, small in sample sizes, and incongruent in conclusions [7-24]. A few meta-analyses [25-33] have also attempted to assess the impacts of ERAS for gastric cancer surgeries, reporting shorter length of stay, reduced hospital cost, and improved recovery of gut function in the ERAS arm, with no increased risk of post-operative complications. Although some studies demonstrated a trend towards an increased risk of readmission, this was not statistically significant and was hence something we were interested to explore. There was significant heterogeneity in the number of included studies (ranging from 6 to 14), attributable to methodological gaps and weaknesses, which we seek to address in this systematic review and meta-analysis.

The aim of this systematic review and meta-analysis is to review the latest body of literature comparing ERAS programs with conventional care in gastric cancer surgery. Unlike previously published meta-analyses, a comprehensive coverage of the literature will be achieved to provide the real-world evidence by including randomized and non-randomized studies in both published and unpublished forms.

\section{Methods}

The study method was conducted as per the guidelines of the Cochrane Handbook of systematic reviews and meta-analysis [25], and reported according to the Preferred Reporting Items for Systematic Reviews and Meta-Analyses (PRISMA) statement guidelines [26]. An electronic search was performed on the following databases: Medline (via PubMed), Embase, OvidSP, Cochrane databases, and the ClinicalTrials.gov website to identify all published and indexed studies reporting gastrectomy outcomes in an enhanced recovery after surgery program. A repetitive and exhaustive combination of the following 'MeSH' search terms were used: "Stomach Neoplasms", "Stomach Diseases", "Stomach", "Gastrectomy", "Laparoscopy", "General Surgery". These were combined with non-MeSH terms including: fast track, ERAS, enhanced recovery after surgery, multimodal, accelerated rehabilitation. The reference lists of relevant studies were manually search to identify additional studies. The last date of search was 10th September 2018. The protocol was registered on PROSPERO prior to the commencement of this study (CRD42017080888).

\section{Inclusion and exclusion criteria}

Studies were included if comparative outcomes were reported for patients undergoing ERAS programs versus conventional care for gastric cancer surgery. There was no restriction on study type and language. However, non-English studies with no extractable data were excluded.

\section{Selection of studies and data extraction}

Two reviewers (IW, NS) independently screened and assessed the studies for inclusion. The studies were first screened by their titles and abstracts. We then retrieved the full-text articles for review if we could not confirm the relevance of the studies for inclusion. If disputed, JS or GK would be the arbiter to resolve differences of opinions regarding the studies' eligibility by discussion and consensus. Full-text articles were retrieved for review if we could not confirm the relevance of the studies for inclusion. The search strategy is shown in the PRISMA diagram (Fig. 1).

Primary outcomes of interest included length of hospital stay (LOS), postoperative morbidity, rates of readmission, time to return of gut function, and total costs. Secondary outcomes of interest included inflammatory markers [C-reactive protein (CRP), interleukin 6 (IL6), tumour necrosis factor alpha $(\mathrm{TNF} \alpha)]$. We abstracted the following data from each study: first author, year, type of publication, age, gender, ERAS measures, length of hospital stay (LOS) in days, rates of postoperative morbidity, rates of readmission, inflammatory markers at postoperative days 1, 3/4, and 7 (CRP, IL6, $\mathrm{TNF} \alpha$ ), time to return of gut function, and total costs.

\section{Data analysis}

All statistical analyses were conducted using RevMan 5.1 software (The Nordic Cochrane Centre, Copenhagen, Denmark). A meta-analysis was conducted to pool weighted mean difference (WMD) or standardized mean difference (SMD) as the summary statistic for continuous variables, and risk ratio (RR) for dichotomous variables. Statistical heterogeneity was assessed using the $I^{2}$ statistic. A fixedeffects model was chosen when the $I^{2}$ statistic value was less than or equal to $50 \%$, and a random-effects model otherwise. Results were reported with 95 percent confidence intervals $(95 \% \mathrm{CI})$, and a $\mathrm{P}$ value of less than 0.05 was considered to be statistically significant. When outcomes were reported as median and range, methods described by Hozo et al. [27] were utilized to convert the values to the mean and standard deviation (SD). In brief, SD was calculated as range/6 and 


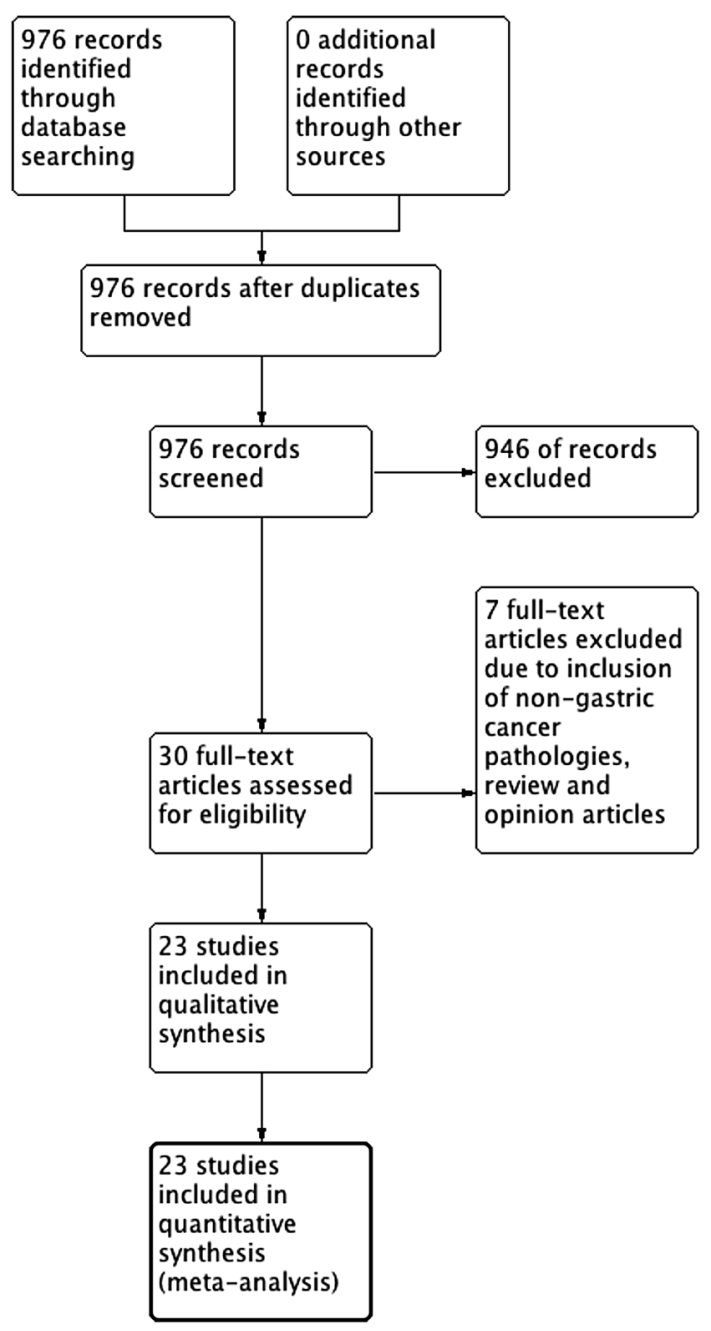

Fig. 1 PRISMA flow diagram

range/4 when the sample sizes were either greater than 70 or between 15 and 59, respectively.

\section{Assessment of bias}

Assessment of study quality and risk of bias for randomized controlled trials was conducted using the Cochrane Risk of Bias tool [25], which included aspects of selection, performance, detection, attrition, reporting and other bias. The Newcastle-Ottawa scale [28] was used to assess quality of non-randomized studies, which included domains of patient selection, comparability of study groups, and outcome assessment. With respect to all aforementioned outcomes of interest, several pooled analyses were conducted and stratified based on the surgical techniques used in the intervention arms. Two subgroup analyses were performed for each endpoint: (1) open versus laparoscopic surgery, (2) high versus low number of items in the ERAS protocol. Based on a published Cochrane review [29] that defined a minimum of
7 out of 17 items in the ERAS protocol as a benchmark, we applied this standard to our study using the consensus guidelines published by the ERAS ${ }^{\circledR}$ Society for gastrectomy [6]. Publication bias was evaluated based on visual inspection for extent of symmetry on the funnel plot, as well as using Egger's regression test if there were more than ten studies.

\section{Results}

\section{Systematic search}

The systematic search revealed a total of 976 publications for possible inclusion. Based on title and abstract review, irrelevant publications, duplicate publications or those not fitting our inclusion criteria were excluded. Thirty publications were reviewed in their entirety and seven were excluded based on the full text, leaving 23 studies included in the final analysis (Fig. 1). This included 14 randomized controlled trials (RCTs) [7-12, 14, 15, 17, 19-21, 23, 30, $31], 5$ prospective cohort studies [16, 18, 22, 32, 33], one of which was a conference abstract [34], and 3 retrospective cohort studies [13, 24].

\section{Study characteristics}

The 23 studies comprised a total of 2686 patients, of which 1391 received ERAS program, and 1295 received conventional care. Six studies [15, 18, 20, 23, 30, 31] reported only the laparoscopic approach, while three studies [14, 16, 23] reported both. For the latter, outcomes were extracted in a stratified manner according to open or laparoscopic, except for one study [16] that did not stratify the outcomes based on surgical technique. Across the studies, the mean age ranged from 52.6 to 80.1 years in the ERAS arm, and 54.5 to 79.6 years in the conventional care arm. The proportion of males ranged from 42.9 to $74 \%$ in the ERAS arm, and $45.5-80 \%$ in the conventional arm. One study [21] stratified the outcomes according to age groups (45-74 years; 75-89 years), and hence was included in the meta-analysis as two separate studies. The characteristics of the included studies are shown in Table 1.

Supplementary table 1 details the ERAS program technical measures reported in all, except for seven studies due to insufficient information in full-text [7-9, 19, 23, 34]. The average number of items reported was 9.7/17. As only one study registered below the benchmark number of ERAS items, we could not perform subgroup analysis assessing high versus low number of ERAS protocol items [13]. None of the studies reported patient adherence rates, hence subgroup analysis investigating patient adherence could not be performed (Supplementary table 1). 


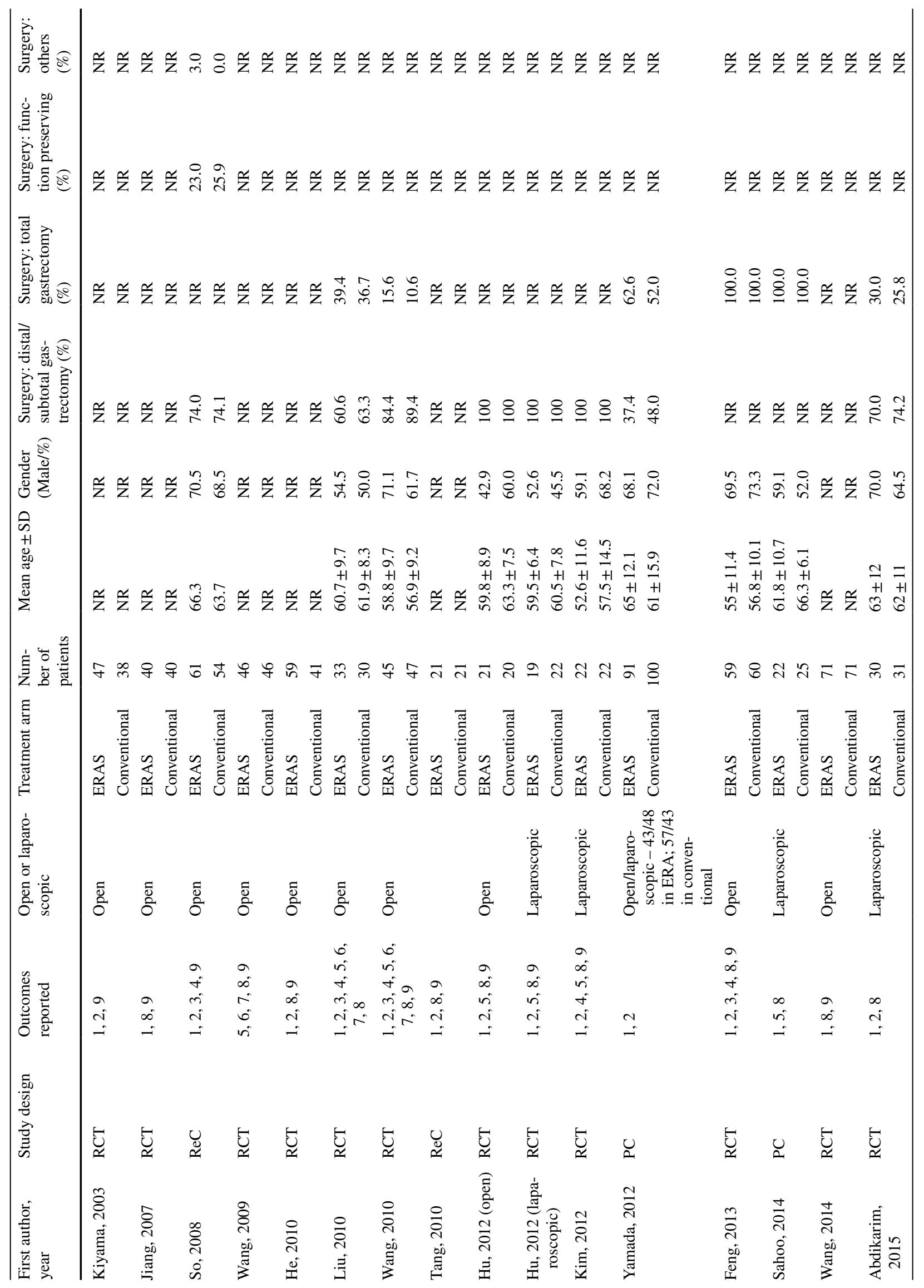




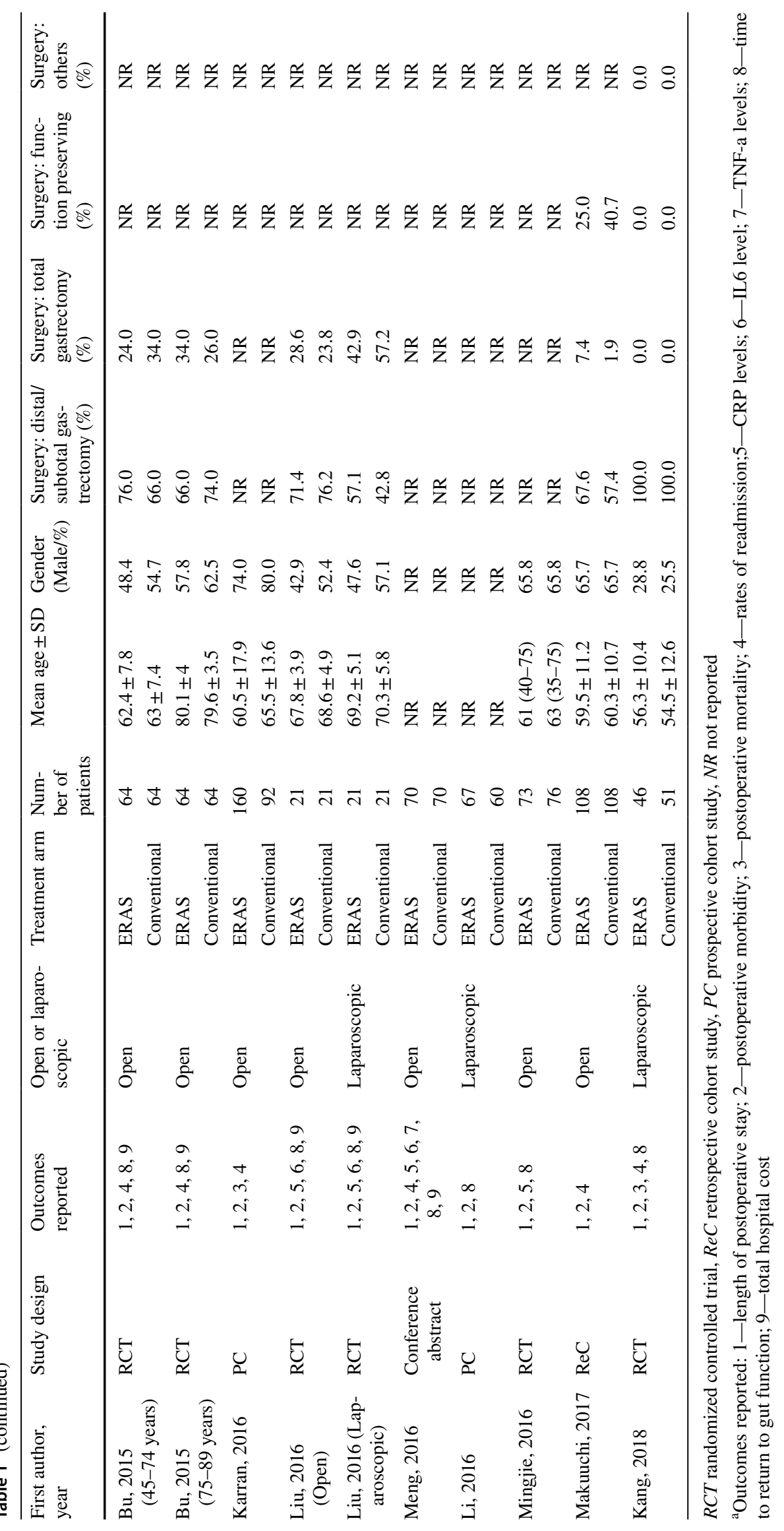




\section{Study quality}

Of the eight cohort studies assessed using the NewcastleOttawa scale [28], five studies [13, 16, 18, 22, 24] had a score above 7 out of the maximum 9, and were deemed to be robust with regards to bias arising from patient selection, comparability of study groups, and outcome assessment. One study scored 5 [23], and one was not assessed due to the limited information in the conference abstract (Supplementary table 2). The risk-of-bias for randomized trials was assessed using the Cochrane Risk-of-Bias tool [25], where all studies had high risk for performance and detection bias as blinding of participants, personnel and/or outcome assessors was not performed in any trial. However, the authors concede that this is an inherent limitation in surgical interventions.

\section{Length of hospital stay (LOS)}

All except one study [9] reported LOS, involving 2469 participants, which yielded a statistically significant shorter LOS in the ERAS arm in the overall analysis (WMD-2.47 days, $95 \% \mathrm{CI}-3.06$ to $-1.89, P<0.00001)$, with significant heterogeneity between the studies $\left(I^{2}=91 \%, P<0.00001\right)$. It was evident graphically from the forest plot that the LOS consistently favored the ERAS arm across individual studies. Subgroup analysis likewise recapitulated the aforementioned advantages, with both open (WMD-2.89 days $95 \%$ $\mathrm{CI}-3.66$ to $-2.12, P<0.00001)$ and laparoscopic (WMD1.70 days, $95 \% \mathrm{CI}-2.63$ to $-0.76, P<0.00001)$ approaches demonstrating shorter LOS in the ERAS arm (Fig. 2). There was a moderate risk of publication bias based on the funnel plot (Figure S1), and Egger's test was significant $(P=0.047)$.

\section{Postoperative morbidity}

Seventeen studies involving 4348 participants evaluated the rate of post-operative morbidity. There were no statistically significant differences in postoperative morbidity between ERAS and conventional care in the overall analysis ( $\mathrm{RR}=0.96,95 \% \mathrm{CI} 0.75-1.23, P=0.73)$, with a low level of heterogeneity between the studies $\left(I^{2}=45 \%\right.$, $P=0.01)$. In subgroup analysis, the laparoscopic approach reported significantly higher morbidity rates in the ERAS $\operatorname{arm}(\mathrm{RR}=1.49,95 \%$ CI 1.04-2.13, $P=0.009)$ (Fig. 3). Although the slight asymmetry in the funnel plot points toward a possible influence of publication bias, Egger's test was not significant for the risk of small-study effects $(P=0.083)$ (Figure S2).

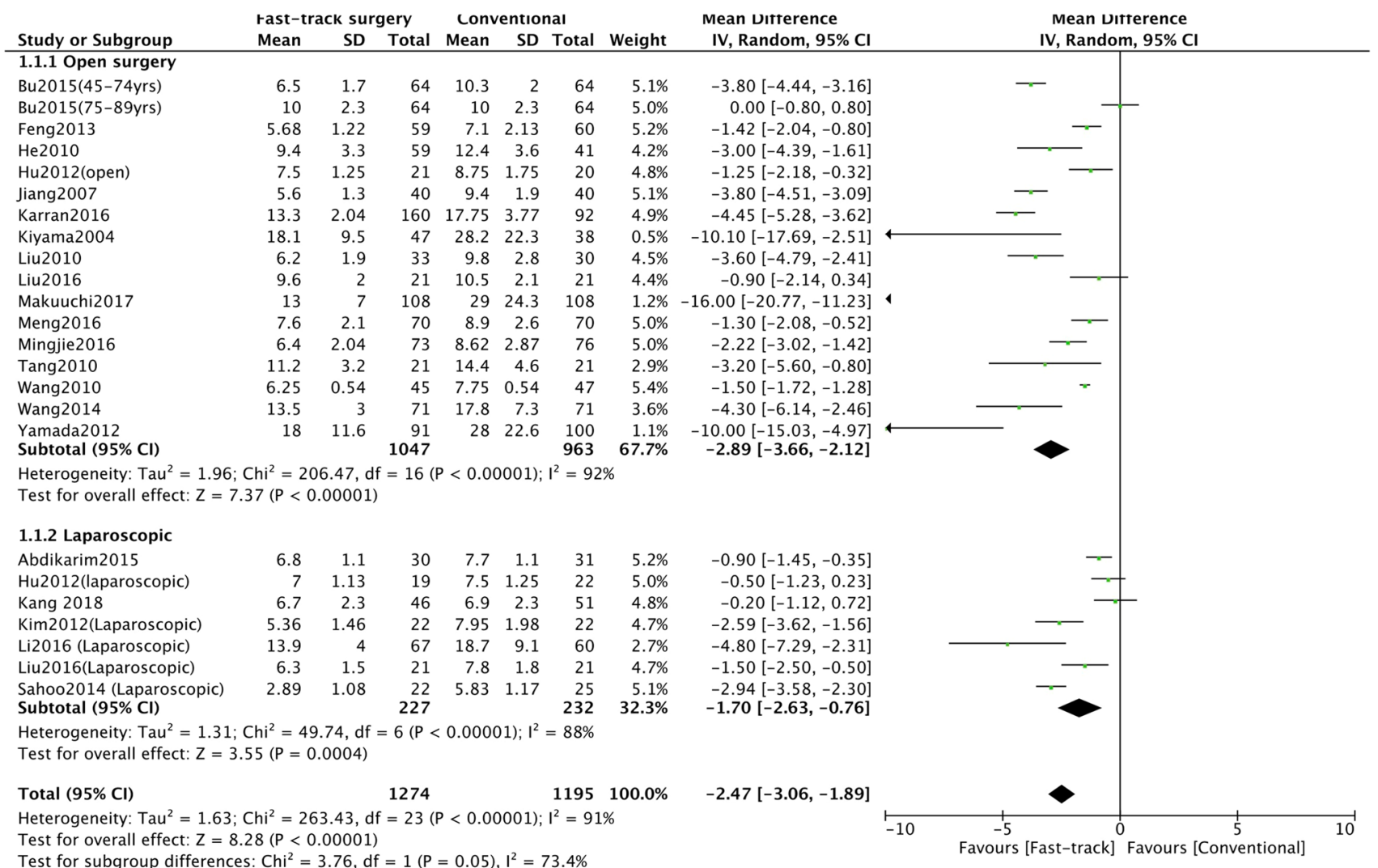

Fig. 2 Length of hospital stay (LOS) 


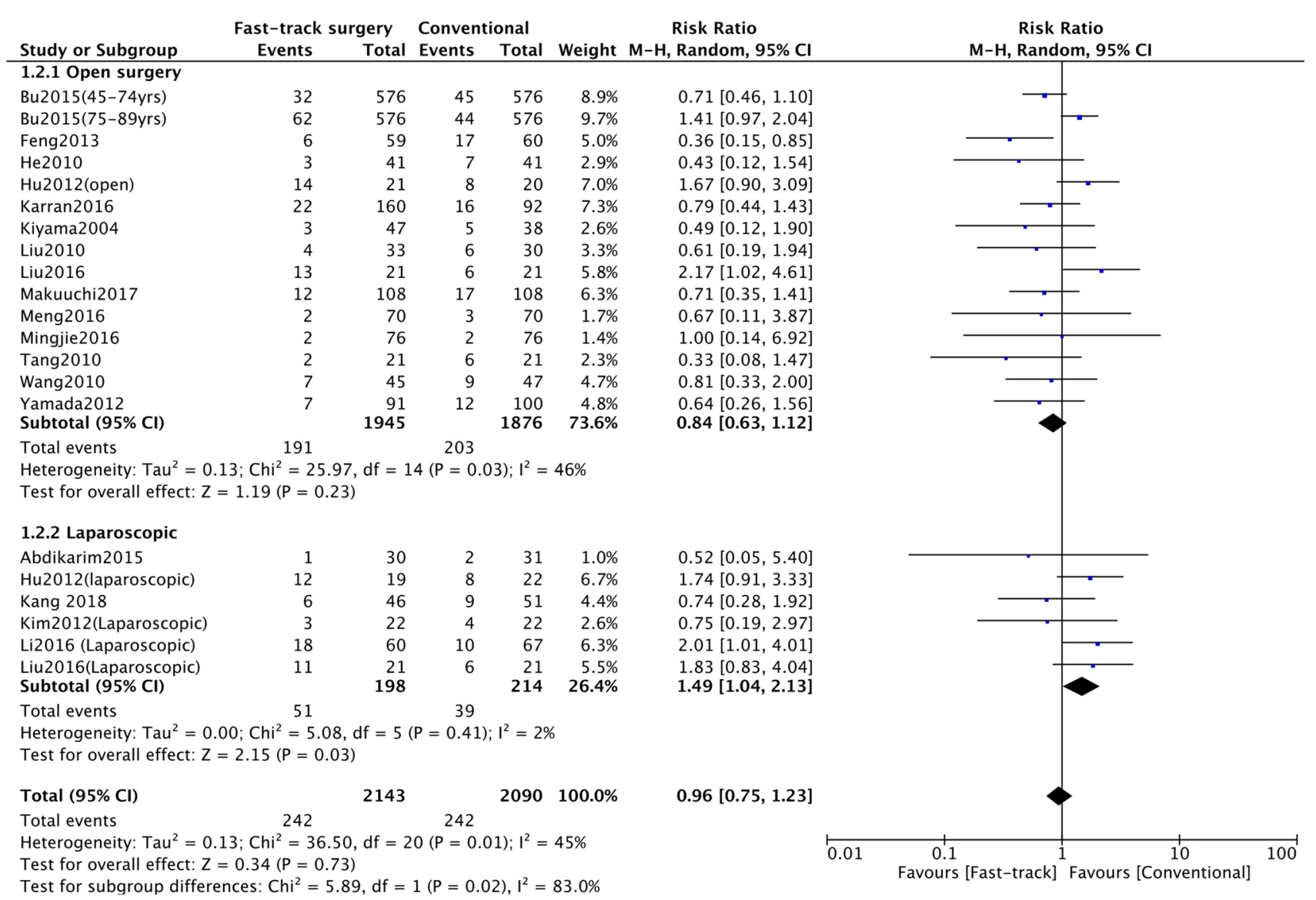

Fig. 3 Postoperative morbidity

\section{Readmission rates}

Nine studies involving 1273 participants reported readmission rates. Intriguingly, there was a statistically significant increase in readmission rates in the ERAS arm than in the conventional arm (RR $=1.95,95 \% \mathrm{CI} 1.03-3.67, P=0.04)$, with no heterogeneity between the studies $\left(I^{2}=0 \%\right.$, $P=0.70)$. This was likewise corroborated in subgroup analysis, where the open approach reported significantly increased readmission rates in the ERAS arm ( $\mathrm{RR}=1.92,95 \% \mathrm{CI}$ 1.00-3.67, $P=0.05$ ) (Fig. 4). There was no evidence of publication bias, evident from the high degree of symmetry in the funnel plot, and Egger' test $(P=0.288)$ (Figure S3).

\section{Time to return of gut function}

Fourteen studies involving 1643 participants reported time to return of gut function. The pooled analysis demonstrated that gut function recovered quicker in the ERAS arm, as evident from the significantly shorter time to return to flatus (WMD -0.70 days, $95 \% \mathrm{CI}-1.02$ to $-0.37, P<0.0001$ ), although statistical heterogeneity across studies was significantly high $\left(I^{2}=96 \%, P<0.00001\right)$. This was reiterated in

the subgroup analysis, where both the open (WMD-0.73 days, $95 \% \mathrm{CI}-1.21$ to $-0.24, P=0.003$ ), and laparoscopic arms (WMD -0.65 days, $95 \%$ CI -0.97 to -0.33 , $P<0.0001)$ reported an improved recovery time in the ERAS arm (Fig. 5). There was no evidence of publication bias, given the high degree of symmetry in the funnel plot, and Egger's test $(P=0.903)$ (Figure S4).

\section{Total cost}

Thirteen studies involving 1358 participants provided data on total cost, yielding a significantly lower cost in the ERAS arm (WMD—4.40, 95\% CI -5.58 to $-3.21, P<0.00001$ ), although statistical heterogeneity was high across studies $\left(I^{2}=83 \%, P<0.00001\right)$. Subgroup analyses recapitulated the aforementioned (Fig. 6). Again, there was no evidence of publication bias, as seen from the high degree of symmetry in the funnel plot, and Egger's test $(P=0.157)$ (Figure S5).

\section{Additional outcomes}

The meta-analysis also examined post-operative inflammatory markers and 30-day mortality. CRP levels were 


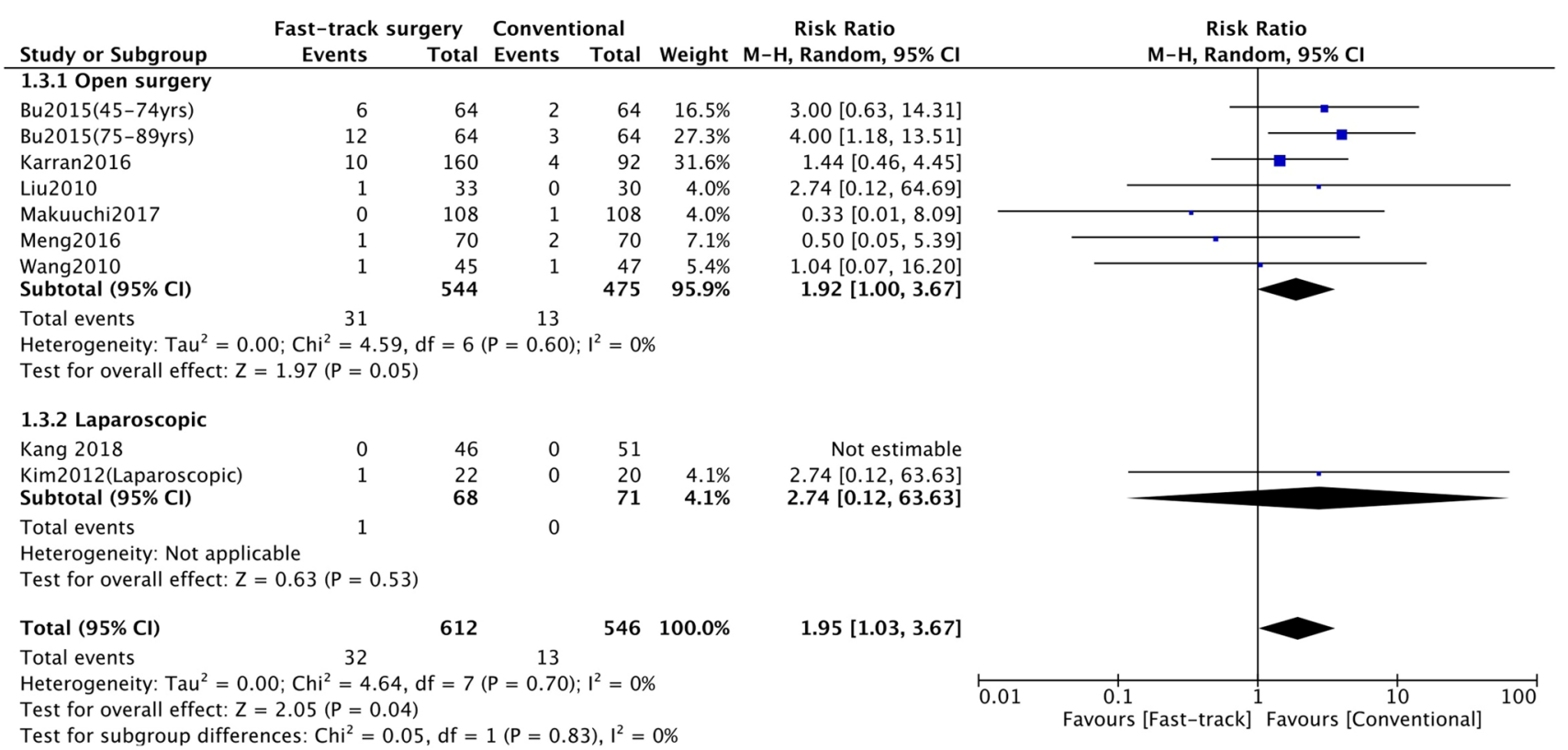

Fig. 4 Readmission rates

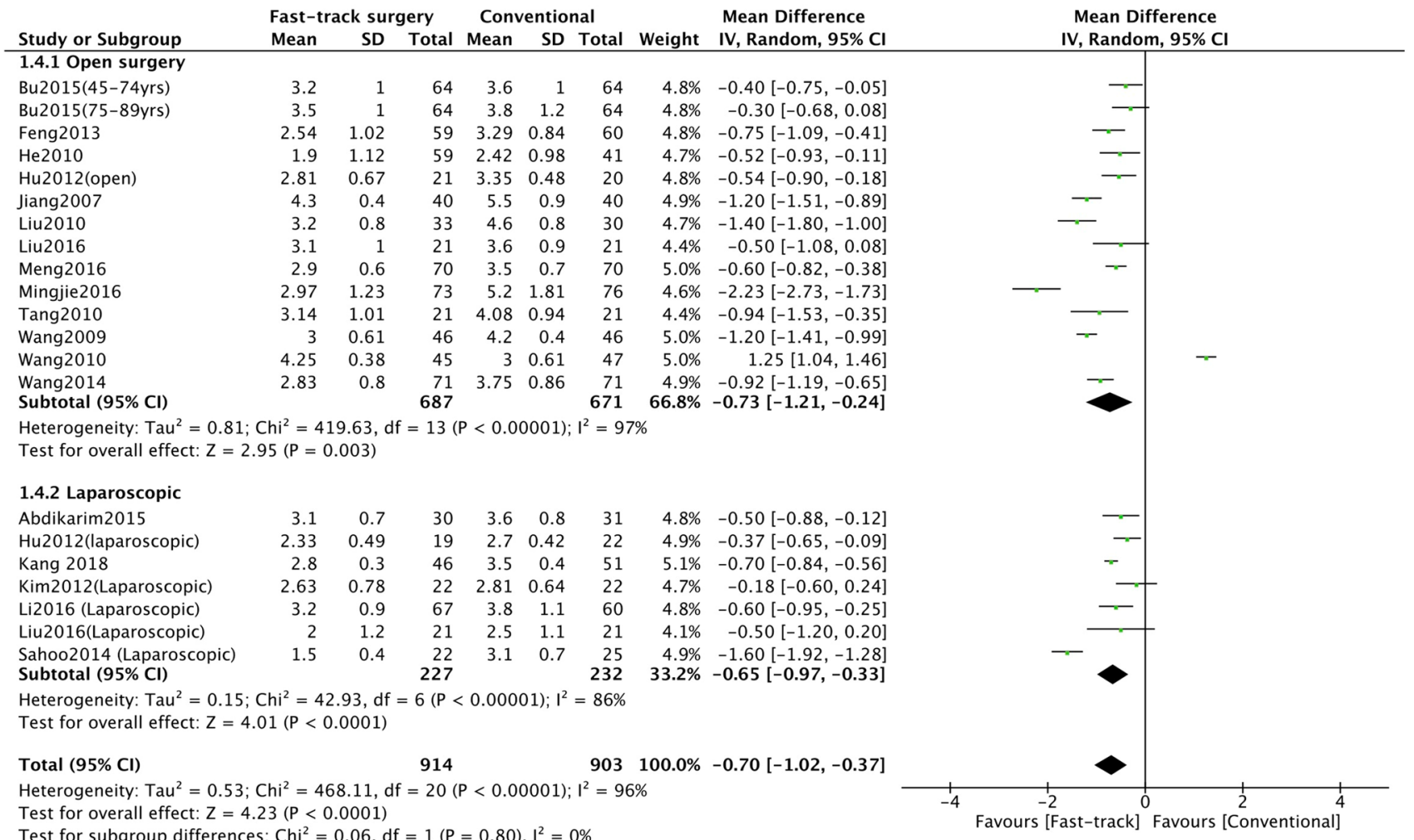

Fig. 5 Time to return of gut function

significantly lower in the ERAS arm on post operative days 3/4 (WMD-22.05 mg/L 95\% CI -28.32 to $-15.78 \mathrm{mg} / \mathrm{L}$, $P<0.00001, N=8$ studies) and $7(\mathrm{WMD}-18.14 \mathrm{mg} / \mathrm{L}$
$95 \% \mathrm{CI}-24.21$ to $-12.07 \mathrm{mg} / \mathrm{L}, P<0.00001, N=6$ studies), but not on day 1 (WMD-11.46 mg/L 95\%CI -28.26 to $-5.34 \mathrm{mg} / \mathrm{L}, P=0.18, N=8$ studies). IL6 levels were 


\begin{tabular}{|c|c|c|c|c|c|c|c|}
\hline \multirow[b]{2}{*}{ Study or Subgroup } & \multicolumn{3}{|c|}{ Fast-track surgery } & \multicolumn{3}{|c|}{ Conventional } & \multirow[b]{2}{*}{ Weight } \\
\hline & Mean & SD & Total & Mean & SD & Total & \\
\hline \multicolumn{8}{|l|}{ 1.5.1 Open surgery } \\
\hline Bu2015(45-74yrs) & 33.5 & 3.4 & 64 & 39.9 & 4 & 64 & $7.8 \%$ \\
\hline Bu2015(75-89yrs) & 42.1 & 4 & 64 & 42.5 & 4.8 & 64 & $7.5 \%$ \\
\hline Feng2013 & 65.2 & 12.4 & 59 & 71.9 & 13.3 & 60 & $3.7 \%$ \\
\hline $\mathrm{He} 2010$ & 29.6 & 4.4 & 59 & 34.6 & 3.4 & 41 & $7.5 \%$ \\
\hline Hu2012(open) & 27.6 & 3 & 21 & 29 & 7 & 20 & $5.1 \%$ \\
\hline Jiang2007 & 18.6 & 2.4 & 40 & 20.4 & 2.4 & 40 & $8.0 \%$ \\
\hline Kiyama2004 & 91.1 & 17.1 & 47 & 117.2 & 47.7 & 38 & $0.5 \%$ \\
\hline Liu2016 & 35.8 & 3.6 & 21 & 40.5 & 2.3 & 21 & $7.1 \%$ \\
\hline Meng2016 & 28.5 & 4.7 & 70 & 35.3 & 7.6 & 70 & $6.8 \%$ \\
\hline Tang2010 & 36.6 & 4.8 & 21 & 45.6 & 7.8 & 21 & $4.4 \%$ \\
\hline Wang2009 & 27.2 & 3.9 & 46 & 31 & 3.6 & 46 & $7.5 \%$ \\
\hline Wang2010 & 44.3 & 6 & 45 & 51.1 & 5.9 & 47 & $6.3 \%$ \\
\hline $\begin{array}{l}\text { Wang2014 } \\
\text { Subtotal }(95 \% \mathrm{Cl})\end{array}$ & 23.8 & 3.7 & $\begin{array}{r}71 \\
628\end{array}$ & 27.8 & 6.1 & $\begin{array}{r}71 \\
603\end{array}$ & $\begin{array}{r}7.3 \% \\
79.5 \%\end{array}$ \\
\hline \multicolumn{8}{|c|}{$\begin{array}{l}\text { Heterogeneity: } \text { Tau }^{2}=5.20 ; \mathrm{Chi}^{2}=82.96, \mathrm{df}=12(P<0.00001) ; \mathrm{I}^{2}=86 \% \\
\text { Test for overall effect: } Z=6.40(P<0.00001)\end{array}$} \\
\hline \multicolumn{8}{|l|}{ 1.5.2 Laparoscopic } \\
\hline Hu2012(laparoscopic) & 33.1 & 2.3 & 19 & 35.8 & 3.1 & 22 & $7.3 \%$ \\
\hline Kim2012(Laparoscopic) & 45.8 & 4.3 & 22 & 47.8 & 5.7 & 22 & $5.5 \%$ \\
\hline $\begin{array}{l}\text { Liu2016(Laparoscopic) } \\
\text { Subtotal }(95 \% \mathrm{CI})\end{array}$ & 33.6 & 2.8 & $\begin{array}{l}21 \\
62\end{array}$ & 38.7 & 1.9 & $\begin{array}{l}21 \\
65\end{array}$ & $\begin{array}{r}7.6 \% \\
20.5 \%\end{array}$ \\
\hline \multicolumn{8}{|c|}{$\begin{array}{l}\text { Heterogeneity: } \mathrm{Tau}^{2}=1.94 ; \mathrm{Chi}^{2}=6.19, \mathrm{df}=2(\mathrm{P}=0.05) ; \mathrm{I}^{2}=68 \% \\
\text { Test for overall effect: } Z=3.52(P=0.0004)\end{array}$} \\
\hline Total $(95 \% \mathrm{Cl})$ & & & 690 & & & 668 & $100.0 \%$ \\
\hline \multicolumn{8}{|c|}{$\begin{array}{l}\text { Heterogeneity: } \mathrm{Tau}^{2}=4.27 ; \mathrm{Chi}^{2}=89.21, \mathrm{df}=15 \\
\text { Test for overall effect: } \mathrm{Z}=7.27(\mathrm{P}<0.00001) \\
\text { Test for subqroup differences: } \mathrm{Chi}^{2}=0.96, \mathrm{df}=1\end{array}$} \\
\hline
\end{tabular}

Fig. 6 Total cost

significantly lower in the ERAS arm on post operative days 1 (SMD-1.57, 95\% CI -2.39 to $-075, P=0.0002$, $N=5$ studies), $3 / 4$ (SMD-1.02, 95\% CI -2.00 to -0.04 , $P=0.04, N=4$ studies), but not 7 (SMD-4.29, 95\% CI -8.99 to $0.40, P=0.07, N=2$ studies). Lastly, TNF $\alpha$ levels was significantly lower in the ERAS arm on days $3 / 4$ (SMD-0.36, 95\% CI -0.61 to $-0.11, P=0.005, N=3$ studies), but not on day 1 (SMD-0.49, $95 \% \mathrm{CI}-1.20$ to $0.23, P=0.18, N=4$ studies) (Figures $\mathrm{S} 6-\mathrm{S} 13$ ). A pooled analysis of 12 studies $(n=1313)$ revealed no significant difference in 30-day mortality between both arms $(\mathrm{RR}=0.58$, 95\% CI 0.06-6.10, $P=0.65$ ) (Figure S14).

\section{Discussion}

We summarized the total body of evidence comparing ERAS program versus conventional care for patients undergoing gastric cancer surgery. Our findings demonstrate that the ERAS program resulted in shorter length of hospital stay (LOS), reduced hospital costs, reduced time to return of gut function, without compromising on risks of morbidity and mortality; however, an increased rate of readmission was observed in the ERAS arm, a finding not demonstrated in previous meta-analyses. Furthermore, there were weaknesses in these studies [35-43]. All except one meta-analysis [38] included only randomized controlled trials (RCTs); Li et al. [37] only examined laparoscopic gastrectomy; Ding et al. [36] limited their analysis to English language articles.

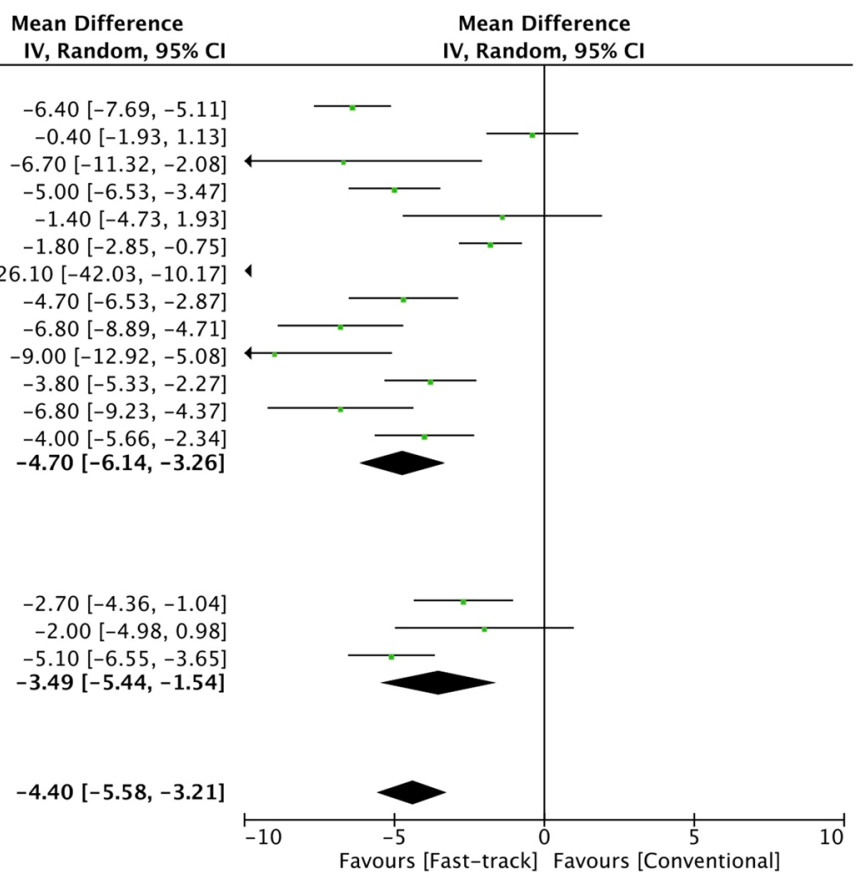

In another study, all surgeries of the gastrointestinal tract were included which portends significant heterogeneity [32]. In addition, there are newer studies that have been published recently. Hence, our study serves as the latest and most comprehensive review of the literature on ERAS for gastric cancer surgery.

Our analysis demonstrated an increased rate of readmission in the ERAS arm, a contentious finding that was also shown by Ding et al. [36]. This trend, albeit non-significant, was also seen in other meta-analyses [37, 38, 41]. As this is contrariwise to the goals of ERAS to attenuate surgical stress and promote recovery, further research is warranted to investigate this antithetical phenomenon. ERAS programs have previously been scrutinized due to the increased risk of readmission particularly in colorectal cancer surgery [44], possibly due to early discharge [45]. However, this concern has not been realized in urological [46], gynaecological [47], and lung surgery [48]. This observed difference could be because gastric cancer surgery is intrinsically a higher-risk surgery, with complication rates reported in the literature to be as high as $45 \%[49,50]$. Late complications, in particular, may have been missed during the initial admission and hence could be the cause of readmission. In addition, gastric cancer patients are generally older, more malnourished and more advanced in stage compared to other cancer patients. Hence, they may have higher risk of readmission after discharge from surgery [2, 41, 44].

Readmissions may be unpredictable [49], however, measures can be employed early to identify postoperative 
complications to mitigate this. For instance, a telephone interview and home-visit can be conducted post-discharge by a nurse clinician; patients can also be reviewed earlywithin a week-at outpatient clinics [32, 50, 51]. Individualized-patient education has also shown benefits compared to a standard education booklet [53-55].

Although risks of post-operative morbidity were similar between both groups, subgroup analysis of patients who underwent laparoscopic surgery revealed an increased risk of morbidity in the ERAS arm. This observation could be attributed to the effects of a learning curve or adaptation phase in some of these studies. Nevertheless, visual inspection of the forest plot suggests inconsistencies between studies, as half were in favor of the ERAS arm whilst the other half were in favor of the conventional care arm.

Nonetheless, the findings from this study should be interpreted in the context of known limitations. Significant statistical heterogeneity was observed in the various outcomes, with the accompaniment of qualitative heterogeneity which we cannot eliminate completely, such as surgeon competency and definition of inclusion criteria. Since the ERAS consensus guidelines for gastrectomy was only published recently [6], a major limitation is the significant heterogeneity of the ERAS protocol in included studies, as these were likely to have been developed from pre-existing ERAS protocols employed in colorectal [4], gynaecology [52], and hepatobiliary [53] surgery. Aside, poor or varied compliance to protocol, as reported in the literature [54], may exacerbate heterogeneity. Next, the findings from this review may not apply to patients who have received neoadjuvant radiotherapy or chemotherapy since they were excluded from most studies. Although these treatment regimens can potentiate malnutrition hence making them unsuitable for ERAS [10, $12,13,17,55]$, ERAS actually involves key preoperative measures that aim to get patients in the best possible condition for surgery. In this regard, one might advocate that patients who received neoadjuvant treatment might actually benefit more from an ERAS regimen and it is therefore questionable whether these patients deserve to be excluded. Furthermore, neoadjuvant or perioperative chemotherapy is the standard of care prior to gastrectomy for cancer in many Asian and Western countries [56-58]. Lastly, as all studies included were from Asia, it may limit the application to Western populations. However, this might be attributed to the skewed global distribution of gastric cancers, where incidence in East Asia is 3-5 times greater than that in Western Europe and North America [59].

Our meta-analysis carries a few other implications for future studies. Firstly, it is notable that ERAS protocols of included studies did not mandate the use of laparoscopic approach, which is otherwise regarded a key constituent in ERAS for colorectal surgery. However, despite encouraging evidence of laparoscopic gastrectomy showing superior short-term outcomes for early gastric cancer and with emerging evidence for advanced gastric cancer [60, 61], it is still important for their effects to be studied methodically in an ERAS setting given the higher morbidity rate in the laparoscopic group. Secondly, the association between ERAS and inflammatory markers is still not well understood. Hence, future trials should attempt to investigate this relationship including TNF $\alpha$ to better inform clinicians and researchers. Thirdly, early initiation of oral feeding maybe controversial in the gastric cancer surgery, which is based on concerns regarding gastric distention and anastomotic leakage. However, most studies in the ERAS arm employed early oral feeding $[11,12,15,17,21,23]$, and our analysis showed that ERAS was associated with faster recovery of gut without increase in morbidity. Finally, future studies should standardize the reporting of outcomes, as well as compliance to the items in the protocol.

\section{Conclusions}

This meta-analysis is the most up-to-date and comprehensive review of the literature. Enhanced recovery after gastric cancer surgery reduces hospital stay, costs, surgical stress response and time to return of gut function as compared to conventional care. However, it may be associated with increased risk of readmission. Caution is necessary when adopting ERAS for gastric cancer surgery.

Funding NLS and IW are supported by Wong Hock Boon Society funds from the Yong Loo Lin School of Medicine, National University of Singapore.

\section{Compliance with ethical standards}

Conflict of interest All authors declare no conflicts of interest.

\section{References}

1. Guggenheim DE, Shah MA. Gastric cancer epidemiology and risk factors. J Surg Oncol. 2013;107(3):230-6.

2. Tegels JJ, De Maat MF, Hulsewe KW, Hoofwijk AG, Stoot JH. Improving the outcomes in gastric cancer surgery. World J Gastroenterol. 2014;20(38):13692-704.

3. Bardram L, Funch-Jensen P, Jensen P, Crawford ME, Kehlet $\mathrm{H}$. Recovery after laparoscopic colonic surgery with epidural analgesia, and early oral nutrition and mobilisation. Lancet. 1995;345(8952):763-4

4. Varadhan KK, Neal KR, Dejong CH, Fearon KC, Ljungqvist O, Lobo DN. The enhanced recovery after surgery (ERAS) pathway for patients undergoing major elective open colorectal surgery: a meta-analysis of randomized controlled trials. Clin Nutr (Edinb, Scotl). 2010;29(4):434-40.

5. Lassen K, Soop M, Nygren J, Cox PB, Hendry PO, Spies C, et al. Consensus review of optimal perioperative care in colorectal 
surgery: Enhanced Recovery After Surgery (ERAS) Group recommendations. Arch Surg (Chic, Ill 1960). 2009;144(10):961-9.

6. Mortensen K, Nilsson M, Slim K, Schafer M, Mariette C, Braga $\mathrm{M}$, et al. Consensus guidelines for enhanced recovery after gastrectomy: Enhanced Recovery After Surgery (ERAS(R)) Society recommendations. Br J Surg. 2014;101(10):1209-29.

7. Kiyama TTT, Yoshiyuki T, Mitsuhashi K, Ise Y, Mizutani T, Okuda T, Fujita I, Masuda G, Kato S, Matsukura N, Tokunaga $\mathrm{A}$, Hasegawa $\mathrm{S}$. Clinical significance of a standardized clinical pathway in gastrectomy patients. J Nippon Med Sch. 2003;70(3):263-9.

8. Jiang ZW, Li JS, Wang ZM, Li N, Liu XX, Li WY, et al. The safety and efficiency of fast track surgery in gastric cancer patients undergoing D2 gastrectomy. Zhonghua wai ke za zhi (Chin J Surg). 2007;45(19):1314-7.

9. Wang DS, Zhou YB, Kong Y, Wang QG, Wang H. Observation of fast track surgery in patients with gastric cancer. Zhonghua wei chang wai ke za zhi (Chin J Gastrointest Surg). 2009;12(5):462-6.

10. He ZG, Tang Y, Wu HG, Wei B, Chen L, Li R. Benefits of perioperative fast-track surgery program on clinical outcome in patients with gastric cancer. Chin J Clin Nutr. 2010;18(1):29-32.

11. Liu XX, Jiang ZW, Wang ZM, Li JS. Multimodal optimization of surgical care shows beneficial outcome in gastrectomy surgery. J Parenter Enter Nutr. 2010;34(3):313-21.

12. Wang D, Kong Y, Zhong B, Zhou X, Zhou Y. Fast-track surgery improves postoperative recovery in patients with gastric cancer: a randomized comparison with conventional postoperative care. J Gastrointest Surg. 2010;14(4):620-7.

13. Tang YWX, Wei B. Clinical application of perioperative fast-track and nutrition support program in elderly patients with gastric cancer. China J Clin Nutr. 2010;18:137-40.

14. chen Hu J, xin Jiang L, Cai L, tao Zheng H, yuan Hu S, bing Chen $\mathrm{H}$, et al. Preliminary experience of fast-track surgery combined with laparoscopy-assisted radical distal gastrectomy for gastric cancer. J Gastrointest Surg. 2012;16(10):1830-9.

15. Kim JW, Kim WS, Cheong JH, Hyung WJ, Choi SH, Noh SH. Safety and efficacy of fast-track surgery in laparoscopic distal gastrectomy for gastric cancer: a randomized clinical trial. World J Surg. 2012;36(12):2879-87.

16. Yamada T, Hayashi T, Cho H, Yoshikawa T, Taniguchi H, Fukushima $\mathrm{R}$, et al. Usefulness of enhanced recovery after surgery protocol as compared with conventional perioperative care in gastric surgery. Gastric Cancer. 2012;15(1):34-41.

17. Feng F, Ji G, Li JP, Li XH, Shi H, Zhao ZW, et al. Fast-track surgery could improve postoperative recovery in radical total gastrectomy patients. World J Gastroenterol. 2013;19(23):3642-8.

18. Sahoo MR, Gowda MS, Kumar TA. Early rehabilitation after surgery program versus conventional care during perioperative period in patients undergoing laparoscopic assisted total gastrectomy. J Minimal Access Surg. 2014;10(3):132-8.

19. Wang G, Yang Y, Zhou B, Chen Y, Jin C, Wang Z, et al. Promotion of postoperative recovery with fast track surgery for gastric cancer patients undergoing gastrectomy: a prospective randomized controlled study. Zhonghua wei chang wai ke za zhi (Chin J Gastrointest Surg). 2014;17(5):489-91.

20. Abdikarim I, Cao XY, Li SZ, Zhao YQ, Taupyk Y, Wang Q. Enhanced recovery after surgery with laparoscopic radical gastrectomy for stomach carcinomas. World J Gastroenterol. 2015;21(47):13339-44.

21. Bu J, Li N, Huang X, He S, Wen J, Wu X. Feasibility of fast-track surgery in elderly patients with gastric cancer. J Gastrointest Surg. 2015;19(8):1391-8.

22. Karran A, Wheat J, Chan D, Blake P, Barlow R, Lewis WG. Propensity score analysis of an enhanced recovery programme in upper gastrointestinal cancer surgery. World J Surg. 2016;40(7):1645-54.
23. Liu G, Jian F, Wang X, Chen L. Fast-track surgery protocol in elderly patients undergoing laparoscopic radical gastrectomy for gastric cancer: a randomized controlled trial. OncoTargets Ther. 2016;9:3345-51.

24. Makuuchi R, Sugisawa N, Kaji S, Hikage M, Tokunaga M, Tanizawa $\mathrm{Y}$, et al. Enhanced recovery after surgery for gastric cancer and an assessment of preoperative carbohydrate loading. Eur J Surg Oncol. 2017;43(1):210-7.

25. GS HJ. Cochrane handbook for systematic reviews of interventions. Chichester: The Cochrane Collaboration and John Wiley \& Sons Ltd; 2008.

26. Moher D, Liberati A, Tetzlaff J, Altman DG. Preferred reporting items for systematic reviews and meta-analyses: the PRISMA statement. Int J Surg (Lond Engl). 2010;8(5):336-41.

27. Hozo SP, Djulbegovic B, Hozo I. Estimating the mean and variance from the median, range, and the size of a sample. BMC Med Res Methodol. 2005;5(1):13.

28. Wells G, Shea B, O'Connell J, Robertson J, et al. The Newcastle-Ottawa Scale (NOS) for assessing the quality of nonrandomised studies in meta-analysis. 2011. http://www.ohri.ca/ programs/clinical_epidemiology/oxford.asp.

29. Spanjersberg WR, Reurings J, Keus F, van Laarhoven CJHM. Fast track surgery versus conventional recovery strategies for colorectal surgery. Cochrane Database Syst Rev. 2011. https:// doi.org/10.1002/14651858.CD007635.pub2.

30. Mingjie X, Luyao Z, Ze T, YinQuan Z, Quan W. Laparoscopic radical gastrectomy for resectable advanced gastric cancer within enhanced recovery programs: a prospective randomized controlled trial. J Laparoendosc Adv Surg Tech Part A. 2017;27(9):959-64.

31. Kang SH, Lee Y, Min SH, Park YS, Ahn SH, Park DJ, et al. Multimodal enhanced recovery after surgery (ERAS) program is the optimal perioperative care in patients undergoing totally laparoscopic distal gastrectomy for gastric cancer: a prospective, randomized, clinical trial. Ann Surg Oncol. 2018;25(11):3231-8.

32. So JB, Lim ZL, Lin HA, Ti TK. Reduction of hospital stay and cost after the implementation of a clinical pathway for radical gastrectomy for gastric cancer. Gastric Cancer. 2008;11(2):81-5.

33. Li Y, Qiu J, Cao H. Application of enhanced recovery after surgery for patients with laparoscopic radical gastrectomy. Zhonghua wei chang wai ke za zhi (Chin J Gastrointest Surg). 2016;19(3):269-73.

34. Meng C, Cao S. Clinical efficacy of enhanced recovery after surgery in the radical gastrectomy for gastric cancer: a prospective study. Surg Endosc Other Interv Tech. 2016;30:485.

35. Li MZ, Wu WH, Li L, Zhou XF, Zhu HL, Li JF, et al. Is ERAS effective and safe in laparoscopic gastrectomy for gastric carcinoma? A meta-analysis. World J Surg Oncol. 2018;16(1):17.

36. Ding J, Sun B, Song P, Liu S, Chen H, Feng M, et al. The application of enhanced recovery after surgery (ERAS)/fast-track surgery in gastrectomy for gastric cancer: a systematic review and metaanalysis. Oncotarget. 2017;8(43):75699-711.

37. Li Z, Wang Q, Li B, Bai B, Zhao Q. Influence of enhanced recovery after surgery programs on laparoscopy-assisted gastrectomy for gastric cancer: a systematic review and meta-analysis of randomized control trials. World J Surg Oncol. 2017;15(1):207.

38. Beamish AJ, Chan DS, Blake PA, Karran A, Lewis WG. Systematic review and meta-analysis of enhanced recovery programmes in gastric cancer surgery. Int J Surg (Lond Engl). 2015;19:46-54.

39. Chen ZX, Liu AH, Cen Y. Fast-track program vs traditional care in surgery for gastric cancer. World J Gastroenterol. 2014;20(2):578-83.

40. Li YJ, Huo TT, Xing J, An JZ, Han ZY, Liu XN, et al. Metaanalysis of efficacy and safety of fast-track surgery in gastrectomy for gastric cancer. World J Surg. 2014;38(12):3142-51. 
41. Yu Z, Zhuang CL, Ye XZ, Zhang CJ, Dong QT, Chen BC. Fast-track surgery in gastrectomy for gastric cancer: a systematic review and meta-analysis. Langenbeck's Arch Surg. 2014;399(1):85-92.

42. Wang LH, Zhu RF, Gao C, Wang SL, Shen LZ. Application of enhanced recovery after gastric cancer surgery: an updated metaanalysis. World J Gastroenterol. 2018;24(14):1562-78.

43. Siotos C, Stergios K, Naska A, Frountzas M, Pergialiotis V, Perrea $\mathrm{DN}$, et al. The impact of fast track protocols in upper gastrointestinal surgery: a meta-analysis of observational studies. Surg J R Coll Surg Edinb Irel. 2018;16(3):183-92.

44. Basse L, Thorbol JE, Lossl K, Kehlet H. Colonic surgery with accelerated rehabilitation or conventional care. Dis Colon Rectum. 2004;47(3):271-7; (discussion 7-8).

45. Kahokehr A, Sammour T, Zargar-Shoshtari K, Thompson L, Hill AG. Implementation of ERAS and how to overcome the barriers. Int J Surg (Lond Engl). 2009;7(1):16-9.

46. Daneshmand S, Ahmadi H, Schuckman AK, Mitra AP, Cai J, Miranda G, et al. Enhanced recovery protocol after radical cystectomy for bladder cancer. J Urol. 2014;192(1):50-5.

47. Myriokefalitaki E, Smith M, Ahmed AS. Implementation of enhanced recovery after surgery (ERAS) in gynaecological oncology. Arch Gynecol Obstet. 2016;294(1):137-43.

48. Rogers LJ, Bleetman D, Messenger DE, Joshi NA, Wood L, Rasburn NJ, et al. The impact of enhanced recovery after surgery (ERAS) protocol compliance on morbidity from resection for primary lung cancer. J Thorac Cardiovasc Surg. 2018;155(4):1843-52.

49. Azimuddin K, Rosen L, Reed JF, Stasik JJ, Riether RD, Khubchandani IT. Readmissions after colorectal surgery cannot be predicted. Dis Colon Rectum. 2001;44(7):942-6.

50. Saunders RS, Fernandes-Taylor S, Rathouz PJ, Saha S, Wiseman JT, Havlena J, et al. Outpatient follow-up versus 30-day readmission among general and vascular surgery patients: a case for redesigning transitional care. Surgery. 2014;156(4):949-56.

51. Nabagiez JP, Shariff MA, Khan MA, Molloy WJ, McGinn JT Jr. Physician assistant home visit program to reduce hospital readmissions. J Thorac Cardiovasc Surg. 2013;145(1):225-33 (discussion 32-33).

52. Kalogera E, Bakkum-Gamez JN, Jankowski CJ, Trabuco E, Lovely JK, Dhanorker S, et al. Enhanced recovery in gynecologic surgery. Obstet Gynecol. 2013;122(2 Pt 1):319-28.

53. Jones C, Kelliher L, Dickinson M, Riga A, Worthington T, Scott MJ, et al. Randomized clinical trial on enhanced recovery versus standard care following open liver resection. Br J Surg. 2013;100(8):1015-24.

54. Ahmed J, Khan S, Lim M, Chandrasekaran TV, MacFie J. Enhanced recovery after surgery protocols - compliance and variations in practice during routine colorectal surgery. Colorectal Dis. 2012;14(9):1045-51.

55. Hu JCJL, Cai L. Preliminary experience of fast-track surgery combined with laparoscopy-assisted radical distal gastrectomy for gastric cancer. J Gastrointest Surg. 2012;16:1,830-1,9..

56. Hosoda K, Azuma M, Katada C, Moriya H, Mieno H, Ishido $\mathrm{K}$, et al. A phase II study of neoadjuvant chemotherapy with docetaxel, cisplatin, and S-1, followed by gastrectomy with D2 lymph node dissection for high-risk advanced gastric cancer: results of the KDOG1001 trial. Gastric Cancer. 2018. https://doi. org/10.1007/s10120-018-0884-0.

57. Tsuburaya A, Mizusawa J, Tanaka Y, Fukushima N, Nashimoto A, Sasako M. Neoadjuvant chemotherapy with S-1 and cisplatin followed by $\mathrm{D} 2$ gastrectomy with para-aortic lymph node dissection for gastric cancer with extensive lymph node metastasis. $\mathrm{Br}$ J Surg. 2014;101(6):653-60.

58. Fava BEC, da Costa WL Jr, Medeiros MLL, Sonagli M, de Castro Ribeiro HS, Diniz AL, et al. Neoadjuvant intraperitoneal chemotherapy followed by radical surgery and HIPEC in patients with very advanced gastric cancer and peritoneal metastases: report of an initial experience in a western single center. World J Surg Oncol. 2018;16(1):62.

59. Torre LA, Bray F, Siegel RL, Ferlay J, Lortet-Tieulent J, Jemal A. Global cancer statistics, 2012. CA Cancer J Clin. 2015;65(2):87-108.

60. Kim W, Kim HH, Han SU, Kim MC, Hyung WJ, Ryu SW, et al. Decreased morbidity of laparoscopic distal gastrectomy compared with open distal gastrectomy for stage i gastric cancer: shortterm outcomes from a multicenter randomized controlled trial (KLASS-01). Ann Surg. 2016;263(1):28-35.

61. Hu Y, Huang C, Sun Y, Su X, Cao H, Hu J, et al. Morbidity and mortality of laparoscopic versus open D2 distal gastrectomy for advanced gastric cancer: a randomized controlled trial. J Clin Oncol. 2016;34(12):1350-7.

Publisher's Note Springer Nature remains neutral with regard to jurisdictional claims in published maps and institutional affiliations. 